\title{
Eigenfunction Expansions for Schrödinger and Dirac Operators with Singular Potentials
}

\author{
G. Nenciu \\ Institute of Atomic Physics, Bucharest, Romania \\ Received October 15, 1974; in revised form February 12, 1975
}

\begin{abstract}
The spectral theory (eigenfunction expansion theorem) is developed for Schrödinger and Dirac operators with classes of potentials including $g \exp (-\mu|\boldsymbol{x}|) /|\boldsymbol{x}|^{2}$ in the Schrödinger case and $g \exp (-\mu|\boldsymbol{x}|) /|\boldsymbol{x}|$ in the Dirac case.
\end{abstract}

\section{Introduction}

In the last decade an extensive literature has been devoted to the spectral and scattering theory for Schrödinger and Dirac operators [1-16] and references therein. One of the most powerful methods in this area is that initiated by lkebe [2]: the generalized eigenfunction expansion method. Although, since the appearence of Ikebe's paper, his proof was generalized and simplified [3-7] it is still rather technical and not general enough to handle singularities like $1 /|\boldsymbol{x}|^{2}$ in the Schrödinger case or $1 /|\boldsymbol{x}|$ in the Dirac case [actually in the Dirac case the Yukava potential, $g \exp (-\mu|\boldsymbol{x}|) /|\boldsymbol{x}|$, is the most interesting from the physical point of view].

The aim of this paper is a further generalization and simplification of the proof of the generalized eigenfunction expansion theorem. As a result, our proof applies both to Schrödinger and Dirac cases with singular potentials. Let us point out that we shall not deal here with the most general case we can handle with our method. The reason is that the proof would loose in simplicity requiring minor but long technical details, while no significant gain of relevance to physics is obtained by relaxing, for example, the exponential fall-off at infinity of the potential up to a $|\boldsymbol{x}|^{-\lambda}$ fall-off as far $\lambda>1$, or allowing space-dimensions greater than three.

Recently, results on the absence of the singular continuous spectrum and the asymptotic completeness for Schrödinger operators with singular potentials have been obtained using other methods by Lavine [9], Pearson and Would [10,11] and Babbitt and Balslev [12]. Actually though Lavine states the results only for locally $L^{p}$ potentials with $p>n / 2$ (which rules out $1 /|\boldsymbol{x}|^{2}$ singularities) it appears that his method requires, besides conditions at infinity, only $|V|^{1 / 2}$ to be $H_{0}^{1 / 2}$ bounded with relative bound less than one. As far as the Dirac case is concerned, we do not know about results of this type for singular potentials. However, a complex and powerful theory has been created by Kuroda, Agmon, Schechter [13-15] which could possible be used also in the Dirac case ${ }^{1}$.

$1 \mathrm{l}$ am grateful to the referee for an extensive comment concerning these points. In particular he pointed out that asymptotic completeness for Schrödinger operators in three dimensions, when $V \in L^{1}$ and $|V|^{1 / 2}$ is $H_{0}^{1 / 2}$ bounded with relative bound less than one follows [4, Lemma IV. 10] from the fact that $(H+E)^{-1}-\left(H_{0}+E\right)^{-1}, E \rightarrow \infty$ is trace-class, by an easy extension of the argument in the proof of Theorem Il.37 in [4] (this fails, however in the Dirac case or for greater space dimensions). 
However the generalized eigenfunction expansion method in the form presented here is more convenient from the physicist's point of view for several reasons: it is conceptually and technically simpler; provides the mathematical setting of the familiar formal manipulations with Born series, Lippmann-Schwinger equations etc; works both in Schrödinger and Dirac case, and, more important, provides explicit formulae, via the generalized eigenfunctions, for the matrix elements of the scattering operator.

Our proof contains the same main steps as the original proof of lkebe. However we changed the proof of each step. First of all we shall show that if $|V|^{1 / 2}$ is $\left|H_{0}\right|^{1 / 2}$ bounded with relative bound less than one, and if $|V|^{1 / 2}$ is $\left|H_{0}\right|^{3 / 2}$ (instead of $\left.\left|H_{0}\right|^{1 / 2}\right)$ compact, then $(H-E)^{-1}$, where $H=H_{0}+V$ in the form sense, is meromorphic in $\mathbb{C} \backslash \sigma\left(H_{0}\right)$. This proves that $\sigma_{\text {ess }}(H) \subset \sigma_{\text {ess }}\left(H_{0}\right)$ and allows to define the generalized eigenfunctions in the usual manner. The second main step is the connection between the generalized eigenfunctions and the resolvent of $H$. Here we shall use a formula obtained from the rearrangement of the Born series, and viewed as a relation between bounded operators both in $L^{2}$ and in some weighted $L^{2}$ spaces. This avoids the use of the Fourier transform of the kernel of $(H-E)^{-1}$ $[2,4]$ which does not work in the Dirac case and for $n \geqq 4$ in the Schrödinger case, as well as the need of truncated potentials used in [5-7]. The final step is the limiting procedure in the formula relating the resolvent and the spectral measure of $H$. Again the original lkebe argument works only for Schrödinger case and $n=3$. As the available methods to overcome this difficulty are rather technical [5-7] we shall give a simple method to obtain the estimates needed to validate the limiting procedure.

\section{The Results}

A. Let $H_{0}$ be the Hamiltonian for a spinless nonrelativistic free particle $\left[1\right.$, p. 297], i.e. the unique selfadjoint extension of $-\Delta$ over $\mathbb{R}^{3}$. Let us remind that $H_{0}$ has only absolutely continuous spectrum $\sigma\left(H_{0}\right)=[0, \infty)$, the generalized eigenfunctions $\phi^{0}(\boldsymbol{p} ; \boldsymbol{x})=(2 \pi)^{-3 / 2} \exp (i \boldsymbol{p} \boldsymbol{x}), \boldsymbol{p} \in \mathbb{R}^{3}$ and its resolvent is an integral operator with the kernel

$$
G_{0}(\boldsymbol{x}, \boldsymbol{y} ; E)=(4 \pi)^{-1} \exp (i \lambda|\boldsymbol{x}-\boldsymbol{y}|) /|\boldsymbol{x}-\boldsymbol{y}| ; \quad \lambda=\sqrt{E}, \quad \operatorname{lm} \lambda \geqq 0 .
$$

For a real function we shall denote by $V,|V|^{1 / 2}, V^{1 / 2}$ the operators of multiplication with $V(\boldsymbol{x}),|V(\boldsymbol{x})|^{1 / 2}$ and [sign $\left.V(\boldsymbol{x})\right]|V(\boldsymbol{x})|^{1 / 2}$ respectively. Let $V(\boldsymbol{x})$ with the property that there exists $\mu>0$ such that $\exp (\mu|\cdot| / 2)|V|^{1 / 2}$ is $H_{0}^{1 / 2}$ bounded with relative bound less than one i.e. $\mathscr{D}\left(\exp (\mu|\cdot| / 2)|V|^{1 / 2}\right) \supset \mathscr{D}\left(H_{0}^{1 / 2}\right)$ and there exist $a<1, b<\infty$ such that for all $f \in \mathscr{D}\left(H_{0}^{1 / 2}\right)$

$$
\left\|\exp (\mu|\cdot| / 2)|V|^{1 / 2} f\right\|^{2} \leqq a\left\|H_{0}^{1 / 2} f\right\|^{2}+b\|f\|^{2} .
$$

Let $H$ be the selfadjoint extension of $H_{0}+V$ in the form sense (see [4, Theorem 11.7]). Our result for the Schrödinger case is contained in:

Theorem 1. (Schrödinger case). Let $V$ satisfying (2.2). Let $E_{\text {a.c. }}$ and $E_{\text {sing. }}$ be the projections on $H_{\text {a.c. }}$ and $H_{\text {sing. }}$ for $H=H_{0}+V$ (in the form sense). Then there exists 
a discrete set $\mathscr{E} \subset \mathbb{R}$, bounded below ${ }^{2}$ and a family of functions $\phi(\boldsymbol{p} ; \boldsymbol{x})$ defined for all $\boldsymbol{p} \in \mathbb{R}^{3}$ with $|\boldsymbol{p}|^{2} \notin \mathscr{E}$ so that

i. For any $f \in L^{2}$

exists.

$$
\hat{f}(\boldsymbol{p})=1 . \text { i.m. } \int f(\boldsymbol{x}) \overline{\phi(\boldsymbol{p} ; \boldsymbol{x})} d \boldsymbol{x}
$$

ii.

$$
\left(E_{\text {a.c. }} f\right)(\boldsymbol{x})=\operatorname{li.m} \int \hat{\hat{f}}(\boldsymbol{p}) \phi(\boldsymbol{p} ; \boldsymbol{x}) d \boldsymbol{p}
$$

(1.i.m. here means $L^{2}$ limit as $R \rightarrow \infty$ and $\eta>0$ of $\left.\int_{|\boldsymbol{p}|<R ;\left|\boldsymbol{p}-\boldsymbol{p}_{0}\right|>\eta \text {, all }\left|\boldsymbol{p}_{0}\right|^{2} \in \mathscr{E}}\right)$.

iii.

$$
\|f\|^{2}=\int|\hat{f}(\boldsymbol{p})|^{2} d \boldsymbol{p}+\left\|E_{\text {sing. }} f\right\|^{2} .
$$

iv. Let $\mathscr{R}=[0, \infty) \backslash\{\mathscr{E} \cap[0, \infty\}$ and $[a, b] \subset \mathscr{R}$. Then

$$
\left\|E_{[a, b]} f\right\|^{2}=\int_{a<|\boldsymbol{p}|^{2}<b}|\hat{f}(\boldsymbol{p})|^{2} d \boldsymbol{p} .
$$

v. $H$ has no singular continuous spectrum.

vi. $f \in \mathscr{D}(H)$ if and only if

and in this case $e^{3}$

$$
\int|\boldsymbol{p}|^{4}|\hat{f}(\boldsymbol{p})|^{2} d \boldsymbol{p}<\infty ; \quad E_{\text {sing. }} f \in \mathscr{D}(H)
$$

$$
(H f)(\boldsymbol{x})=\text { l.i.m. } \int|\boldsymbol{p}|^{2} \hat{f}(\boldsymbol{p}) \phi(\boldsymbol{p} ; \boldsymbol{x}) d \boldsymbol{p}+\left(E_{\text {sing. }} H f\right)(\boldsymbol{x}) .
$$

Remarks. 1. By a well known inequality [1; p.307] if

$$
|V(\boldsymbol{x})| \leqq g \exp (-\mu|\boldsymbol{x}|) /|\boldsymbol{x}|^{2}, \quad g<1 / 4
$$

then $V$ satisfies the conditions of the Theorem 1.

2. Let $V(x) \geqq 0$ and $\exp (-\mu|\cdot| / 2) V^{1 / 2}$ be $H_{0}^{1 / 2}$ bounded. Then formulae (3.5), (3.6) below are valid so that Theorem 1 extends to this class of potentials, in particular for potentials satisfying

$$
0 \leqq V(\boldsymbol{x}) \leqq g \exp (-\mu|\boldsymbol{x}|) /|\boldsymbol{x}|^{2}, \quad g<\infty .
$$

3. Although we stated and we shall prove Theorem 1 only for three dimensional case, the generalisation to $n \geqq 4$ presents only minor technical difficulties in proving that $\left[|V|^{1 / 2}\left(H_{0}+1\right)^{-2}|V|^{1 / 2}\right]$ is compact [19].

4. For the sake of simplicity we imposed an exponential fall-off of the potential at infinity. It is a matter of handling the Hölder and Young inequalities and use of Theorem IV.31 in [4] in proving that the Theorem 1 (and also Theorem 2 below) remains true (with the difference that $\mathscr{E}$ is no longer discrete but only closed and of zero measure, and therefore the existence of singular continuous spectrum is not ruled out) for potentials for which $(1+|\cdot|)^{1+\varepsilon}|V|^{1 / 2}$ is $H_{0}^{1 / 2}$ bounded with relative bound less than one.

${ }^{2}$ Actually it can be proved that $E$ is finite.

${ }^{3}$ In fact if $\phi_{n}(x)$ is the complete set of orthonormal eigenfunctions, $f_{n}=\left(f, \phi_{n}\right)$ then

$$
E_{\text {sing. }} f=\sum_{n} f_{n} \phi_{n} ; \quad H E_{\text {sing. }} f=\sum_{n} E_{n} f_{n} \phi_{n} .
$$


B. Let $\left(L^{2}\right)^{4}$ be the Hilbert space of the $\mathbb{C}^{4}$-valued functions $\Psi(\boldsymbol{x}) \equiv\left(\psi_{i}(\boldsymbol{x})\right)_{i=1}^{4}$ with the usual scalar product

$$
(\Psi, \Phi)=\sum_{i=1}^{4} \int \psi_{i}(\boldsymbol{x}) \overline{\varphi_{i}(\boldsymbol{x})} d \boldsymbol{x} .
$$

The Hamiltonian $H_{0}$ of a free Dirac particle of unit mass is given by the selfadjoint extension in $\left(L^{2}\right)^{4}$ of the following differential operator [1]

$$
\alpha P+\beta ; \quad \alpha=\left(\begin{array}{ll}
0 & \sigma \\
\sigma & 0
\end{array}\right), \quad \beta=\left(\begin{array}{rr}
1_{2} & 0 \\
0 & -1_{2}
\end{array}\right)
$$

where $\sigma=\left(\sigma_{1}, \sigma_{2}, \sigma_{3}\right)$ are the Pauli $2 \times 2$ matrices, $1_{2}$ is the $2 \times 2$ unit matrix and

$$
\alpha P=-i \sum_{k=1}^{3} \alpha_{k} \frac{\partial}{\partial x_{k}} .
$$

$H_{0}$ has only absolute continuous spectrum

$$
\sigma\left(H_{0}\right)=(-\infty,-1] \cup[1, \infty)
$$

and its generalized eigenfunctions are

$$
\Phi_{0}^{i, \pm}(\boldsymbol{p} ; \boldsymbol{x})=(2 \pi)^{-3 / 2} u^{i, \pm}(\boldsymbol{p}) \exp (i \boldsymbol{p} \boldsymbol{x}) ; \quad i=1,2,
$$

where

and

$$
u^{i, \pm}(\boldsymbol{p})=[(\varepsilon(\boldsymbol{p}) \pm 1) / 2 \varepsilon(\boldsymbol{p})]^{1 / 2}\left(\begin{array}{c}
\chi_{i} \\
\frac{\sigma \boldsymbol{p}}{1 \pm \varepsilon(\boldsymbol{p})} \chi_{i}
\end{array}\right) ; \quad \chi_{1}=\left(\begin{array}{l}
1 \\
0
\end{array}\right) ; \quad \chi_{2}=\left(\begin{array}{l}
0 \\
1
\end{array}\right)
$$

$$
\varepsilon(\boldsymbol{p})=\left(|\boldsymbol{p}|^{2}+1\right)^{1 / 2} \text {. }
$$

The integral kernel of the resolvent is given by

where

$$
G_{0}(\boldsymbol{x}, \boldsymbol{y} ; E)=\left(\alpha P_{\boldsymbol{x}}+\beta+E\right)(4 \pi)^{-1} \exp (i \lambda|\boldsymbol{x}-\boldsymbol{y}|) /|\boldsymbol{x}-\boldsymbol{y}|,
$$

$$
\lambda^{2}=E^{2}-1, \quad \operatorname{lm} \lambda \geqq 0 .
$$

Theorem 2. (Dirac case). Let $V(x)$ be a $4 \times 4$ hermitean matrix and $V$ the operator defined by

$$
(V \Psi)_{i}(x)=\sum_{j=1}^{4} V_{i j}(x) \psi_{j}(x) ; \quad i=1,2,3,4 .
$$

Let $V$ satisfying the conditions

$D_{1}$ There exists $\mu>0$ such that $\exp (\mu|\cdot| / 2)|V|^{1 / 2}$ is $\left|H_{0}\right|^{1 / 2}$ bounded with relative bound less than one.

$D_{2} \exp (\mu|\cdot|) V_{i j}(\cdot) \in L^{2}$.

Let $E_{\text {a.c. }}$ and $E_{\text {sing. }}$ be the projections on the absolute continuous respectively singular subspaces of $H=H_{0}+V$ in the form sense [17]. Then there exists a 
discrete set $\mathscr{E} C(-\infty,-1] \cup[1, \infty)$ and a family of functions $\Phi^{i, \pm}(\boldsymbol{p} ; \boldsymbol{x})$ defined for all $\boldsymbol{p} \in \mathbb{R}^{3}, \pm \varepsilon(\boldsymbol{p}) \notin \mathscr{E}$ such that

i. For any $\Psi \in\left(L^{2}\right)^{4}$

exists.

$$
\hat{\hat{\psi}}_{i, \pm}(\boldsymbol{p})=\text { l.i.m. } \int \sum_{j=1}^{4} \psi_{j}(\boldsymbol{x}) \overline{\Phi_{j}^{i, \pm}(\boldsymbol{p} ; \boldsymbol{x})} d \boldsymbol{x} ; \quad i=1,2
$$

ii.

$$
\left(E_{\text {a.c. }} \Psi\right)(\boldsymbol{x})=\text { l.i.m. } \int \sum_{i, \pm} \hat{\hat{\psi}}_{i, \pm}(\boldsymbol{p}) \Phi^{i, \pm}(\boldsymbol{p} ; \boldsymbol{x}) d \boldsymbol{p} \text {. }
$$

iii.

$$
\|\Psi\|^{2}=\sum_{i, \pm} \int\left|\hat{\hat{\psi}}_{i, \pm}(\boldsymbol{p})\right|^{2} d \boldsymbol{p}+\left\|E_{\text {sing. }} \Psi\right\|^{2} .
$$

iv. a. Let $\mathscr{R}=\sigma\left(H_{0}\right) \backslash \mathscr{E}$ and $[a, b] \subset \mathscr{R} \cap[1, \infty)$.

Then

$$
\left\|E_{[a, b]} \Psi\right\|^{2}=\sum_{i} \int_{a \leqq \varepsilon(\boldsymbol{p}) \leqq b}\left|\hat{\hat{\psi}}_{i,+}(\boldsymbol{p})\right|^{2} d \boldsymbol{p} .
$$

b. Let $[a, b] \subset \mathscr{R} \cap(-\infty,-1]$. Then

$$
\left\|E_{[a, b]} \Psi\right\|^{2}=\sum_{i} \int_{-b \leqq \varepsilon(\boldsymbol{p}) \leqq-a}\left|\hat{\hat{\psi}}_{i,-}(\boldsymbol{p})\right|^{2} d \boldsymbol{p} .
$$

v. H has no singular continuous spectrum.

vi. $\Psi \in \mathscr{D}(H)$ if and only if

and in this case

$$
\sum_{i, \pm} \int \varepsilon^{2}(\boldsymbol{p})\left|\hat{\hat{\psi}}_{i, \pm}(\boldsymbol{p})\right|^{2} d \boldsymbol{p}<\infty ; \quad E_{\text {sing. }} \Psi \in \mathscr{D}(H)
$$

$$
(H \Psi)(\boldsymbol{x})=\left(\text { l.i.m. } \sum_{i, \pm} \int \pm \varepsilon(\boldsymbol{p}) \hat{\hat{\psi}}_{i, \pm}(\boldsymbol{p}) \Phi^{i, \pm}(\boldsymbol{p} ; \boldsymbol{x}) \mathrm{d} \boldsymbol{p}\right)+\left(E_{\text {sing. }} H \Psi\right)(\boldsymbol{x}) .
$$

Remarks. 5. Again a known inequality [1, p. 307] shows that if

$$
\||V(\boldsymbol{x}) \|| \leqq v \exp (-\mu|\boldsymbol{x}|) /|\boldsymbol{x}|, \quad 0 \leqq v<2 / \pi,
$$

where $\|V(\boldsymbol{x})\| \mid$ is the usual $4 \times 4$ matrix norm then $V$ satisfies the conditions of the Theorem 2. However we expect that potentials satisfying (2.26) with $0 \leqq v<1$ to be physically acceptable. But for $v \geqq 2 / \pi$ even. the definition of the Hamiltonian (the construction of a distinguished self-adjoint extension) is a nontrivial problem [18]. In [19] we shall show that the theory of perturbation by quadratic forms can be extended to provide distinguished self-adjoint extensions for potentials satisfying (2.26) with $v<1$ for which the method of this paper applies.

6. Theorem 2 is true without the condition $D_{2}$ on the potential. We added $D_{2}$ to simplify the proof of compactity of $\left[|V|^{1 / 2} H_{0}^{-2}|V|^{1 / 2}\right][19]$.

\section{Proof of Theorem 1}

As stated in the introduction, we shall give the detailed proof only for Theorem 1 leaving the reader to rewrite the proof for the Dirac case. The proof proceeds in a series of points

1. Let $L_{\gamma}^{2}, \gamma \geqq 0^{4}$

$$
L_{2}^{\gamma}=\left\{f \mid(1+|\cdot|)^{-\gamma} f \in L^{2}\right\}, \quad\|f\|_{\gamma}=\left\|(1+|\cdot|)^{-\gamma} f\right\| .
$$

4 The use of $L_{\gamma}^{2}$ is not new in spectral theory of Schrödinger operator. See for example [16]. 
Of course $L_{0}^{2}=L^{2}$. We shall consider operators defined by the kernels

$$
T(E)=|V|^{1 / 2}\left(H_{0}-E\right)^{-1} V^{1 / 2} ; \quad\left(H_{0}-E\right)^{-1} V^{1 / 2} ; \quad|V|^{1 / 2}\left(H_{0}-E\right)^{-1} .
$$

We shall denote by $T_{\gamma}(E) \equiv[T(E)]_{\gamma},\left[\left(H_{0}-E\right)^{-1} V^{1 / 2}\right]_{\gamma}$ etc. the operators defined in $L_{\gamma}^{2}$ by $T(E),\left(H_{0}-1\right)^{-1} V^{1 / 2}$ etc. Taking into account the factor $\exp (-\mu|x| / 2)$, we refer to $[4$, p.17 and 37$]$ for the proof of the following statements

a. $T_{\gamma}(E) ;\left[|V|^{1 / 2}\left(H_{0}-E\right)^{-1}\right]_{\gamma} ;\left[\left(H_{0}-E\right)^{-1} V^{1 / 2}\right]_{\gamma}$ are bounded operator valued functions of $E$ analytic in $\mathbb{C} \backslash \sigma\left(H_{0}\right)$.

b.

$$
\varlimsup_{E \rightarrow-\infty}\left\|T_{\gamma}(E)\right\| \leqq a^{2}<1 .
$$

2. $\left[\left(H_{0}-E\right)^{-1}\right]_{\gamma}$ is a bounded operator valued function analytic in $\mathbb{C} \backslash \sigma\left(H_{0}\right)$ $\left|\left(\left(H_{0}-E\right)^{-1} f\right)(\boldsymbol{x})\right| \leqq \int(\exp (-\operatorname{lm} \lambda|\boldsymbol{x}-\boldsymbol{y}|) /|\boldsymbol{x}-\boldsymbol{y}|)|f(\boldsymbol{y})| d \boldsymbol{y}$ $\leqq \int_{|\boldsymbol{y}|>2|\boldsymbol{x}|}(\exp (-\operatorname{lm} \lambda|\boldsymbol{x}-\boldsymbol{y}| / 2) /|\boldsymbol{x}-\boldsymbol{y}|)\left((1+|\boldsymbol{y}|)^{-\gamma}|f(\boldsymbol{y})| / \exp (\operatorname{lm} \lambda|\boldsymbol{y}| / 4)(1+|\boldsymbol{y}|)^{-\gamma}\right) d \boldsymbol{y}$ $+(1+|2 \boldsymbol{x}|)^{\gamma} \int_{|\boldsymbol{y}|<2|\boldsymbol{x}|}\left(\exp (-\operatorname{lm} \lambda|\boldsymbol{x}-\boldsymbol{y}|)|f(\boldsymbol{y})| /|\boldsymbol{x}-\boldsymbol{y}|(1+|\boldsymbol{y}|)^{\gamma}\right) d \boldsymbol{y}$ $\leqq\left[(1+|2 \boldsymbol{x}|)^{\gamma}+\sup _{\mathbb{R}^{3}} \frac{(1+|\boldsymbol{y}|)^{\gamma}}{\exp (\operatorname{lm} \lambda|\boldsymbol{y}| / 4)}\right] \int\left(\exp (-\operatorname{lm} \lambda|\boldsymbol{x}-\boldsymbol{y}| / 2)|f(\boldsymbol{y})| /|\boldsymbol{x}-\boldsymbol{y}|(1+|\boldsymbol{y}|)^{\gamma}\right) d \boldsymbol{y}$.

3. Taking into account (3.3) the following operator is well defined for sufficiently negative $E$

$$
R_{\gamma}(E)=\left[\left(H_{0}-E\right)^{-1}\right]_{\gamma}-\left[\left(H_{0}-E\right)^{-1} V^{1 / 2}\right]_{\gamma}\left[1+T_{\gamma}(E)\right]^{-1}\left[|V|^{1 / 2}\left(H_{0}-E\right)^{-1}\right]_{\gamma} .
$$

We refer again to [4, the begining of the proof of Theorem 11.34] for the proof of the fact that for $E \rightarrow-\infty$

$$
R_{0}(E)=(H-E)^{-1} .
$$

4. Let us consider the following operator

$$
\begin{aligned}
\hat{T}_{\gamma}(E)= & T_{\gamma}(E)-T_{\gamma}\left(E_{0}\right)=\left(E-E_{0}\right)\left[|V|^{1 / 2}\left(H_{0}-E\right)^{-1}\left(H_{0}-E_{0}\right)^{-1} V^{1 / 2}\right]_{\gamma} ; \\
& \operatorname{lm} \lambda_{0}>\mu / 2 .
\end{aligned}
$$

Then in the variable $\lambda, \hat{T}_{\gamma}(E)$ is a compact operator valued function analytic in $\operatorname{lm} \lambda>-\mu / 2$. For, let us remark that (2.2) implies that $|V|^{1 / 2} \in L^{2}$. [This can be seen taking in (2.2), $f(x)=\exp (-\mu|x| / 2)]$.

On the other hand

$$
\begin{aligned}
&\left|G_{0}(\boldsymbol{x}, \boldsymbol{y} ; E)-G_{0}\left(\boldsymbol{x}, \boldsymbol{y} ; E_{0}\right)\right| \leqq\left(\exp (-\operatorname{lm} \lambda \mid \boldsymbol{x}-\boldsymbol{y} 0 / 4 \pi)\left|\left(1-\exp \left[i\left(\lambda-\lambda_{0}\right)|\boldsymbol{x}-\boldsymbol{y}|\right]\right) /\right| \boldsymbol{x}-\boldsymbol{y}||\right. \\
& \leqq\left|\lambda-\lambda_{0}\right| \exp (-\operatorname{lm} \lambda|\boldsymbol{x}-\boldsymbol{y}|) / 4 \pi \text { for } \operatorname{lm} \lambda>-\mu / 2
\end{aligned}
$$

and for $\alpha>0$

$$
\exp (\alpha|x-y|) \leqq \exp (\alpha|x|) \cdot \exp (\alpha|y|) .
$$

Introducing (3.8), (3.9) in (3.7) it results that actually $\hat{T}_{\gamma}(E)$ is Hilbert-Schmidt.

5. Let now $\gamma_{0}>3 / 2$ be fixed. Then for $\gamma=0, \gamma_{0},\left[1+T_{\gamma}(E)\right]^{-1}$, in the variable $\lambda$ is meromorphic for $\operatorname{lm} \lambda>-\mu / 2$. 
For, let $E_{0}<0$ such that $\operatorname{lm} \lambda_{0}>\mu / 2$

$$
\max \left\{\left\|T_{0}\left(E_{0}\right)\right\|,\left\|T_{\gamma_{0}}\left(E_{0}\right)\right\|\right\}<1 \text {. }
$$

Then for $\gamma=0, \gamma_{0}$

$$
\begin{aligned}
1+T_{\gamma}(E) & =1+T_{\gamma}\left(E_{0}\right)+\tilde{T}_{\gamma}(E) \\
& =\left(1+T_{\gamma}\left(E_{0}\right)\right)\left(1+\left(1+T_{\gamma}\left(E_{0}\right)\right)^{-1} \tilde{T}_{\gamma}(E)\right) .
\end{aligned}
$$

Now $\left(1+T_{\gamma}\left(E_{0}\right)\right)^{-1}$ is bounded and $\tilde{T}_{\gamma}(E)$ is compact, so that the moromorphy of $\left[1+T_{\gamma}(E)\right]^{-1}$ follows from a well-known result about analytic families of compact operators $[4,20]$.

6. Let for $\gamma=0, \gamma_{0}$

and

$$
\mathscr{E}_{\gamma}=\left\{t \in \mathbb{R} \mid t=\operatorname{Re} E ;\left[1+T_{\gamma}(E)\right]^{-1} \text { does not exist }\right\}
$$

$$
\mathscr{E}=\mathscr{E}_{0} \cup \mathscr{E}_{\gamma_{0}}
$$

From the previous point $\mathscr{E}$ is a discrete set (actually it can be proved that $\mathscr{E}$ is finite).

7. Because $\gamma_{0}>3 / 2, \phi^{0}(\boldsymbol{p} ; \boldsymbol{x})$ are $L_{\gamma_{0}}^{2}$-valued functions of $\boldsymbol{p}$, uniformly continuous in $\mathbb{R}^{3}$.

8. We shall define the generalized eigenfunctions $\phi(p, E ; \cdot) ; p \in \mathbb{R}^{3}, \operatorname{lm} E>0$, $\operatorname{Re} E \notin \mathscr{E}$ as elements of $L_{\gamma_{0}}^{2}$ by

$\phi(\boldsymbol{p}, E ; \cdot)=\phi^{0}(\boldsymbol{p} ; \cdot)-\left[\left(H_{0}-E\right)^{-1} V^{1 / 2}\right]_{\gamma_{0}}\left[1+T_{\gamma_{0}}(E)\right]^{-1}|V|^{1 / 2} \phi^{0}(\boldsymbol{p} ; \cdot)$.

9. Let $\eta>0$ and

$$
\mathscr{F}_{a, b, c}=\{E \mid \operatorname{Re} E \in[a, b] \subset[0, \infty) \backslash \mathscr{E} ; \operatorname{Im} E \in[0, c], c>0\} .
$$

Then $\exp (-\eta|\cdot|) \phi(\boldsymbol{p}, E ; \cdot)$ is a $L_{\gamma_{0}}^{2}$-valued function of $\boldsymbol{p}$ and $E$ which has a uniformly continuous extension on $\mathbb{R}^{3} \times \mathscr{F}_{a, b, c,}$. This follows from (3.14), 5.7. and the fact that $\left[\exp (-\eta|\cdot|)\left(H_{0}-E\right)^{-1} V^{1 / 2}\right]_{\gamma_{0}}$ is a bounded analytic function for $\operatorname{lm} \lambda>\max \{-\eta,-\mu / 2\}$. The last assertion can be easily proved using the (generalized) Hölder [21, p. 527] and Young [22, p.8] inequalities.

10. It is easy to verify that for $\sigma \notin \sigma\left(H_{0}\right)$

$$
\left[\left(H_{0}-E\right)^{-1}\right]_{\gamma_{0}} \phi^{0}(\boldsymbol{p} ; \cdot)=\left(|\boldsymbol{p}|^{2}-E\right)^{-1} \phi^{0}(\boldsymbol{p} ; \cdot)
$$

11. From (3.14), (3.16) for $E \in \mathscr{F}_{a, b, c}, \operatorname{lm} E>0$

$$
R_{\gamma_{0}}(E) \phi^{0}(\boldsymbol{p} ; \cdot)=\left(|\boldsymbol{p}|^{2}-E\right)^{-1} \phi(\boldsymbol{p}, E ; \cdot) .
$$

12. We are able now to make the connection between $(H-E)^{-1}$ and $\phi(p, E ; \cdot)$. Let $\psi(\boldsymbol{x}) \in C_{0}^{\infty}$. We shall define

$$
\hat{\psi}(\boldsymbol{p}, E)=\int \psi(\boldsymbol{x}) \overline{\phi(\boldsymbol{p}, E ; \boldsymbol{x})} d \boldsymbol{x} .
$$

It follows from 9. that $\hat{\psi}(\boldsymbol{p}, E)$ is a uniformly continuous function of $\boldsymbol{p}$ and $E$ on $\mathbb{R}^{3} \times \mathscr{F}_{a, b, c}$. Let $E \in \mathscr{F}_{a, b, c}, \operatorname{lm} E>0$. Then, as $\psi \in L^{2}$, from (3.6)

$$
\begin{aligned}
(H-E)^{-1} \psi & =R_{\gamma_{0}}(E) \psi=R_{\gamma_{0}}(E) \int \hat{\psi}(\boldsymbol{p}) \phi^{0}(\boldsymbol{p} ; \cdot) d \boldsymbol{p}=\int \hat{\psi}(\boldsymbol{p}) R_{\gamma_{0}}(E) \phi^{0}(\boldsymbol{p} ; \cdot) d \boldsymbol{p} \\
& =\int\left(|\boldsymbol{p}|^{2}-E\right)^{-1} \hat{\psi}(\boldsymbol{p}) \phi(\boldsymbol{p}, E ; \cdot) d \boldsymbol{p}
\end{aligned}
$$


where ^ stands for Fourier transform. The integrals in (3.19) are understood as Bochner integrals in $L_{\gamma_{0}}^{2}$ whose existence as well as the possibility of introducing $R_{\gamma_{0}}(E)$ under the integral sign is assured by standard results about Bochner integral [23]. Let now $\varphi(x) \in C_{0}^{\infty}$

$$
\begin{aligned}
\left((H-\bar{E})^{-1} \psi, \varphi\right) & =\left(\psi,(H-E)^{-1} \varphi\right)=\int\left\{\psi(\boldsymbol{x}) \int\left(|\boldsymbol{p}|^{2}-\bar{E}\right)^{-1} \overline{\hat{\varphi}(\boldsymbol{p})} \overline{\phi(\boldsymbol{p}, E ; \boldsymbol{x})} d \boldsymbol{p}\right\} d \boldsymbol{x} \\
& =\left(|\boldsymbol{p}|^{2}-\bar{E}\right)^{-1} \hat{\psi}(\boldsymbol{p}, E) \overline{\hat{\varphi}(\boldsymbol{p})} d \boldsymbol{p}
\end{aligned}
$$

which leads to

$$
\overline{(H-\bar{E})^{-1} \psi}(\boldsymbol{p}, E)=\left(|\boldsymbol{p}|^{2}-\bar{E}\right)^{-1} \hat{\psi}(\boldsymbol{p}, E) .
$$

The interchange of integrals in (3.20) is permitted because $\psi, \varphi \in C_{0}^{\infty}$.

13. From (3.21)

$$
\left\|(H-\bar{E})^{-1} \psi\right\|^{2}=\left.\int|| \boldsymbol{p}\right|^{2}-\left.\bar{E}\right|^{-2}|\hat{\psi}(\boldsymbol{p}, E)|^{2} d \boldsymbol{p} .
$$

14. From this point the proof of the Theorem 1 is identical with the final parts of the proofs of Lemma IV 29 and Theorem IV.25 in [4] and will be not reproduced here.

In the Dirac case where $1 / \varepsilon^{2}(p) \notin L^{1}$ and also in the Schrödinger case for $n \geqq 4$ we need a better estimation on $\hat{\psi}(p, E)$. Again we shall write, for the sake of simplicity, the estimation for the Schrödinger case. Let $\psi \in C_{0}^{\infty}$. The relation (3.21) can be written as

$$
\overline{\left(H_{0}-E_{1}\right)^{-1}\left(H_{0}-\bar{E}\right)(H-\bar{E})^{-1} \psi}(\boldsymbol{p}, E)=\left(|\boldsymbol{p}|^{2}-E_{1}\right)^{-1} \hat{\psi}(\boldsymbol{p}, E),
$$

where $\operatorname{lm} E_{1}>0$. On the other hand it is easy to see that

$$
\left[\left(H_{0}-E\right)^{-1} V^{1 / 2}\right]_{\gamma}=\left[\left(H_{0}-E\right)^{-1}\left(H_{0}-E_{1}\right]_{\gamma}\left[\left(H_{0}-E_{1}\right)^{-1} V^{1 / 2}\right]_{\gamma}\right.
$$

and from (3.5), (3.6) we have

$$
\begin{aligned}
& \left(H_{0}-E_{1}\right)^{-1}\left(H_{0}-\bar{E}\right)(H-\bar{E})^{-1} \\
& \quad=\left(H_{0}-E_{1}\right)^{-1}-\left[\left(H_{0}-E_{1}\right)^{-1} V^{1 / 2}\right]_{0}\left[1+T_{0}(\bar{E})\right]^{-1}\left[|V|^{1 / 2}\left(H_{0}-\bar{E}\right)^{-1}\right]_{0} .
\end{aligned}
$$

It follows from (3.23) and (3.25)

$$
\begin{aligned}
\sup _{E \in \mathscr{F}_{a, b, c}} & \int\left|\left(|\boldsymbol{p}|^{2}-E_{1}\right)\right|^{-2}|\hat{\psi}(\boldsymbol{p}, E)|^{2} d \boldsymbol{p} \leqq 2\left\|\left(H_{0}-E_{1}\right)^{-1} \psi\right\|^{2} \\
& +2\left\|\left[\left(H_{0}-E_{1}\right)^{-1} V^{1 / 2}\right]_{0}\right\|^{2} \\
& \cdot \sup _{E \in \mathscr{F}_{a, b, c}}\left\|\left[1+T_{0}(\bar{E})\right]^{-1}\right\|^{2} \sup _{E \in \mathscr{F}_{a, b, c}}\left\|\left[|V|^{1 / 2}\left(H_{0}-\bar{E}\right)^{-1}\right] \psi\right\|^{2}<\infty .
\end{aligned}
$$

\section{References}

1. Kato, T.: Perturbation theory for linear operators. Berlin-Heidelberg-New York: Springer 1966

2. Ikebe, T.: Arch. Rational Mech. Anal. 5, 1-34 (1960)

3. Thoe,D.W.: Arch. Rational Mech. Anal. 26, 335-356 (1967)

4. Simon, B.: Quantum mechanics for Hamiltonians defined as quadratic forms. Princeton: Princeton University Press 1971 
5. Asholm, P., Schmidt, G.: Arch. Rational Mech. Anal. 40, 281-311 (1971)

6. Thomson, M.: Quart. J. Math. Oxford II Ser. 23, 17-55 (1972)

7. Thomson, M. : Comm. Pure Appl. Math. 25, 499-532 (1972)

8. Prosser, R. T.: J. Math. Phys. 4, 1048-1054 (1963)

9. Lavine, R.: J. Functional Analysis 12, 30-54 (1973)

10. Pearson, D. B., Whould, D.: Nuovo Cimento 14A, 765-780 (1973)

11. Pearson, D. B.: Helv. Phys. Acta 47, 249-265 (1974)

12. Babitt,D., Balslev, E.: U.C.LA (preprint)

13. Agmon, S.: Ann. Pisa (to appear)

14. Kuroda, S. T.: J. Math. Soc. Jap. (to appear)

15. Schechter, M.: Com. Math. Helv. 49, 84-106 (1973)

16. Kato, T., Kuroda, S. T. : Rocky Mountain Math. J. 1, 127-172 (1971)

17. Faris, W. G. : Helv. Phys. Acta 45, 1074-1088 (1972)

18. Kalf,H., Schmincke,U.-W., Walter,J., Wüst, R.: On the spectral theory of Schrödinger and Dirac operators with singular potentials. Symposion on Spectral Theory and Differential Equations. Dundee 1974

19. Nenciu, G.: Relativistic quantum mechanics for Hamiltonians defined as quadratic forms. I. Self-adjointness and invariance of the essential spectrum for Dirac operators. To be published 20. Hunziker, W.: Helv. Phys. Acta 39, 451 - 462 (1966)

21. Dunford, N., Schwartz, J.: Linear operators. I. New York: Interscience Publishers 1958

22. Neri, U.: Singular integrals. Berlin-Heidelberg-New York: Springer 1971

23. Hille,E., Phillips, R.S.: Functional analysis and semigroups. Providence R. I. Amer. Math. Soc. Colloq. Publ. Vol. 31, 1957

Communicated by W. Hunziker

\author{
G. Nenciu \\ Laboratory of Theoretical Physics \\ Institute of Atomic Physics \\ P.O. Box 5206 \\ Bucharest, Romania
}


